\title{
Superconductor-proximity effect in hybrid structures: Fractality versus Chaos
}

\author{
Alexander Ossipov ${ }^{1,2}$ and Tsampikos Kottos ${ }^{1,3}$ \\ ${ }^{1}$ Max-Planck-Institut für Strömungsforschung, Bunsenstraße 10, D-37073 Göttingen, Germany \\ ${ }^{2}$ ICTP, Condensed Matter Section, Strada Costiera 11, I-34014 Trieste, Italy \\ ${ }^{3}$ Hellenic Army, KEחB Thivas, $5 A^{\text {th }}$ Artillery Unit, 32200 Thiva, Greece
}

\begin{abstract}
We study the proximity effect of a superconductor to a normal system with fractal spectrum. We find that there is no gap in the excitation spectrum, even in the case where the underlying classical dynamics of the normal system is chaotic. An analytical expression for the distribution of the smallest excitation eigenvalue $E_{1}$ of the hybrid structure is obtained. On small scales it decays algebraically as $\mathcal{P}\left(E_{1}\right) \sim E_{1}^{-D_{0}}$, where $D_{0}$ is the fractal dimension of the spectrum of the normal system. Our theoretical predictions are verified by numerical calculations performed for various models.
\end{abstract}

PACS numbers: 74.45.+c, 05.45.Mt, 73.23.-b

During the last years there was considerable interest for the study of the statistical properties of hybrid normal-superconductor structures. One of the main outcomes of these studies was the prediction that a normal system in the proximity of a superconductor acquires characteristics that are typical of the superconducting state. Specifically, if the underlying classical dynamics of the normal system is chaotic, an energy gap in the quasiparticle density of states emerges above the Fermi energy. The mean value $\left\langle E_{1}\right\rangle$ of the gap was found [1, 2, 3] to be proportional to the Thouless energy,

$$
\left\langle E_{1}\right\rangle \propto E_{T}=G \delta / 4 \pi=M T \delta / 4 \pi
$$

where $\langle\cdots\rangle$ indicates an ensemble average, $M$ is the number of transverse modes of the point contact between the superconductor and the normal system, $T$ is the tunnel probability per mode and $\delta \sim 1 / L^{d}$ is the mean level spacing of the $d$-dimensional normal system of linear size $L$. The product $G=M T$ is the point contact conductance in units of $2 e^{2} / h$. As a matter of fact, in a recent publication 2] the statistical fluctuations of the lowest excited state around the mean-field value $\left\langle E_{1}\right\rangle$ were studied in the framework of the Random Matrix Theory. It was found that the gap distribution is a universal function of the rescaled energy $x=\left(E_{1}-\left\langle E_{1}\right\rangle\right) / \Delta_{g}$, in a broad range $|x| \ll M^{2 / 3}$. The width of the gap distribution $\Delta_{g} \sim M^{1 / 3} \delta$ is parametrically smaller than the gap size $\left\langle E_{1}\right\rangle$ but bigger than the mean level spacing $\delta$ of the normal system. In contrast, normal systems with integrable classical dynamics do not possess any gap near the Fermi energy. Instead, their density of states vanishes linearly near the Fermi level. Thus, it was naturally proposed, that the appearance or not of a gap in the excitation spectrum of a normal system in the proximity with a superconductor can be used in the studies of quantum chaos as a measure for distinguishing classically chaotic systems from integrable ones. Apart from the two extreme cases discussed above, a fairly good understanding of the proximity gap was obtained also for the generic case of systems with mixed classical phase space [4]. In this case it was found that the excitation gap reduces below the value of fully chaotic systems (11).

The investigation of the proximity gap has recently been extended to quantum systems in the diffusive regime [5] where it was found that a similar type of gap appears. The value of $E_{1}$ is given by Eq. (11) provided that we substitute the ballistic conductance $M T$ with the appropriate expression $D L^{2-d}$ for diffusive systems. Here $D$ is the classical diffusion coefficient.

Despite all this activity, a significant class of systems was left unexplored. Namely hybrid structures whose normal part has fractal spectra. The latter exhibit energy level statistics that are in strong contrast to the level repulsion predicted by Random Matrix Theory (RMT) [6]. Their level spacing distribution follows inverse power laws $P(s) \sim s^{-\beta}$ which is a signature of level clustering. The power $\beta$ was found to be related with the fractal dimension of the spectrum $D_{0}$ as $\beta=1+D_{0}$ []]. Realizations of this class are quasi-periodic systems with metalinsulator transition at some critical value of the on-site potential like the Harper model [7, 8], Fibonacci chains 7, 9], or quantum systems with chaotic classical limit as the Kicked Harper Model 10, 11, 12]. Apart of their own interest the analysis of these systems can be illuminating for the understanding of the behavior of high dimensional disordered systems at the metal-insulator transition like the $3 d$ Anderson model [13].

Here, for the first time, we present consequences of the fractal nature of the spectrum of the normal system in the excitation spectrum of the hybrid structure. We consider the normal system connected to the superconductor via point contacts supporting $M$ channels and show that there is no gap in the excitation spectrum even in the case where the corresponding classical phase space is chaotic (like in the case of the Kicked Harper model). We derive an analytical expression for the distribution of the minimum excitation eigenvalue $\mathcal{P}\left(E_{1}\right)$, and show that its behavior is dictated by the fractal dimension $D_{0}$ of the 
spectrum of the normal part. Thus the nature of the classical dynamics becomes totally irrelevant for these type of systems. Specifically we show that $\mathcal{P}\left(E_{1}\right)$ generated over different Fermi energies behaves as

$$
\mathcal{P}\left(\tilde{E}_{1}\right)=\left(\frac{1}{D_{0}}-1\right)\left(\tilde{E}_{1}^{-D_{0}}-1\right)
$$

where $\tilde{E}_{1}=2 \frac{E_{1}}{s_{\max }}$ and $s_{\max }$ is the maximum level spacing of the normal system within the energy interval that is used in order to generate statistics. Eq. (2) is the main outcome of our investigation. A consequent result is that the mean $\tilde{E}_{1}$ is given by

$$
\left\langle\tilde{E}_{1}\right\rangle=\frac{1-D_{0}}{2-D_{0}}
$$

One has to note the lack of any dependence on the system size $L$ and the number of channels $M$ in contrast to Eq.(11). Our theoretical results (23) are confirmed by numerical calculations performed for various systems with fractal spectra.

Let us start our analysis with the Kicked Harper (KH) model. The system is defined by the time depended Hamiltonian

$$
H=Q \cos (p)+K \cos (\theta) \sum_{m} \delta(t-m T)
$$

where $p$ denotes the angular momentum, $\theta$ the conjugate angle, while the kick period is $T$. Contrary to the standard Harper model (corresponding to the limit $K \rightarrow 0$ ) this model for large enough $K \geq 5$ is classically chaotic.

The quantum mechanics of this system is described by a time evolution operator for one period

$$
U=\exp \left(-i \frac{Q}{\hbar} \cos (\hbar \hat{p})\right) \exp \left(-i \frac{K}{\hbar} \cos (x)\right)
$$

where $\hat{p}=-i d / d \theta$ is the momentum operator and $\hbar$ is an effective Planck constant, which includes the frequency ratio of the unperturbed system and the external driving. For $K=Q$ the quasi-energy spectrum is fractal 10,11 . and we always consider cases where $\hbar / 2 \pi$ is strongly irrational. Using a recently proposed recipe 3] we can write down the corresponding quantum Andreev map $\mathcal{F}$ and find the quasi-energies of the excitation spectrum of the hybrid structure by direct diagonalization of $\mathcal{F}$. In all cases considered bellow we have generated more than 3000 values of $E_{1}$ for statistical processing.

Figure 1 shows $\mathcal{P}\left(E_{1}\right)$ for various system sizes $L$ and number of channels $M$. For small values of $E_{1}$ the distribution of the minimum excitation eigenvalue displays clearly an inverse power law. Moreover, it is independent of the number of channels and system size in agreement with Eq. (2). In the insets of Fig. 1 we also report our results for the mean value of $E_{1}$ for various system sizes and various numbers of channels. The inverse power law character of the distribution $\mathcal{P}\left(E_{1}\right)$ forces us to conclude that

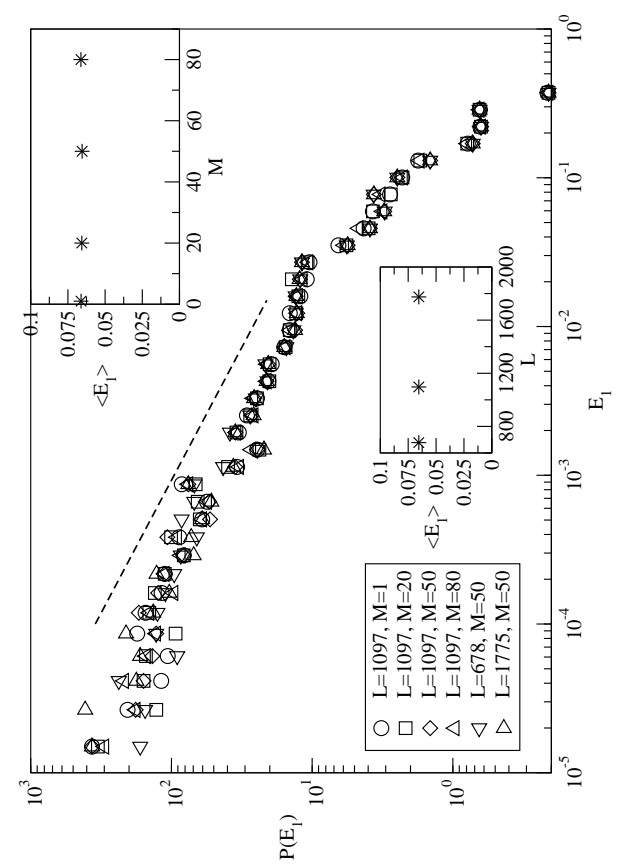

FIG. 1: The distribution of the lowest excited energy, generated over an ensemble of different Fermi energies, for the KH model (4). Various symbols correspond to different system sizes and numbers of channels. All data overlap with one another indicating that $\mathcal{P}\left(E_{1}\right)$ is insensitive to the number of channels and the system size. The dashed line is the theoretical prediction of Eq. (2). In the insets we show the numerically evaluated $\left\langle E_{1}\right\rangle$ versus the system size $L$ and the number of channels $M$.

the probability to find a quasi-energy excitation smaller than $\left\langle E_{1}\right\rangle$ is high and thus there is no gap in the excitation spectrum (even in a probabilistic sense).

The validity of Eqs. 23) can be verified in more cases in the Fibonacci chain model of a one dimensional quasicrystal where various scaling exponents $D_{0}$ can be obtained. The normal system is described by the tightbinding Hamiltonian:

$$
H=\sum_{n}|n\rangle V_{n}\langle n|+\sum_{n}(|n\rangle\langle n+1|+| n+1\rangle\langle n|)
$$

where $V_{n}$ is the potential at site $n$. It only takes the two values $+V$ and $-V$ arranged in a Fibonacci sequence [9]. It was shown that the spectrum is a Cantor set with zero Lebesgue- measure for all $V>0$. The sample is in contact with $M$ semi-infinite one-dimensional superconductors which are attached in $M$ randomly chosen sites. The quasi-energy spectrum of the hybrid structure is calculated by employing the effective Hamiltonian approach 1]. We again find inverse power laws for the distributions $\mathcal{P}\left(E_{1}\right)$. Here the exponent depends on the potential strength $V$, while Eq. (2) still relates the resulting 


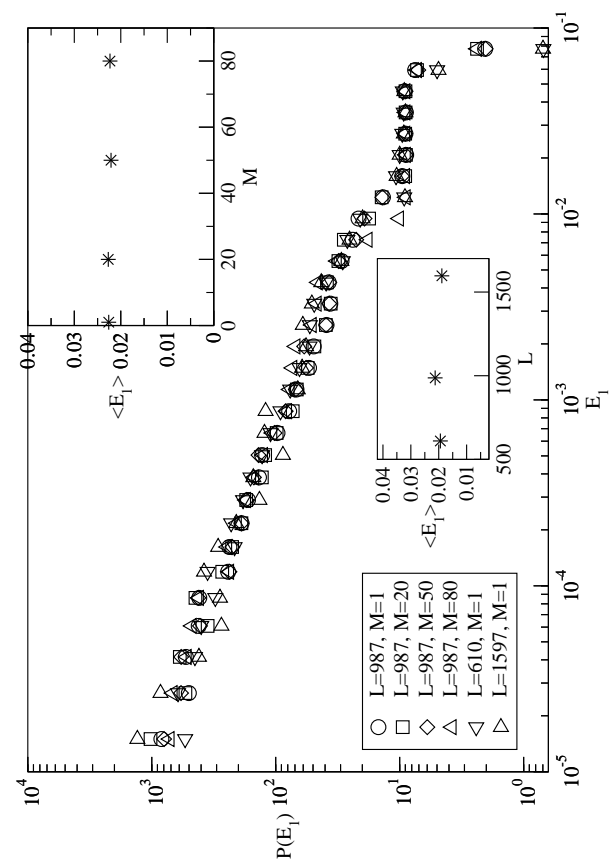

FIG. 2: The distribution $\mathcal{P}\left(E_{1}\right)$ of a hybrid structure consisting of a a Fibonacci sample of size $L$ (normal part) attached with $M$ semi-infinite one-dimensional superconductors. The data for various $L, M$ fall one on top of the other, indicating that $\mathcal{P}\left(E_{1}\right)$ is independent of $L, M$. In the insets we report the mean value $\left\langle E_{1}\right\rangle$ of the distribution as a function of system size $L$ and number of channels $M$.

statistics to the fractal dimension $D_{0}^{E}$.

In Fig. 2 we report our results for $\mathcal{P}\left(E_{1}\right)$ for $V=1.4$ and various system sizes $L$ and number of channels $M$. In the insets we also plot the mean value $\left\langle E_{1}\right\rangle$ of the distribution. Similarly with Fig. 1 we observe that $\mathcal{P}\left(E_{1}\right)$ and consequently $\left\langle E_{1}\right\rangle$ are independent of $L$ and $M$. In Fig. 3 we summarize our results for various $V$ values. A nice agreement between our numerical data and the theoretical predictions (23) is observed [14].

The above results call for a theoretical explanation. Our starting point is the observation that the distribution $\mathcal{P}\left(E_{1}\right)$ rescaled in appropriate way is the same for the normal and the hybrid structure. This assumption is verified in Fig. 4 where we plot the distribution of the minimum excitation level $\tilde{E}_{1}$ for a representative case where the normal part is a Fibonacci lattice with $V=1.4$. The overlap between the resulting distributions of the hybrid structure and of the corresponding normal system is evident. In order to understand this phenomenon one should recall that the eigenstates of the normal system are not extended like typical chaotic eigenstates, but they have fractal structure. As a result, most of the eigenstates have intensities at the boundary with a superconductor which are so small that one can consider that they are
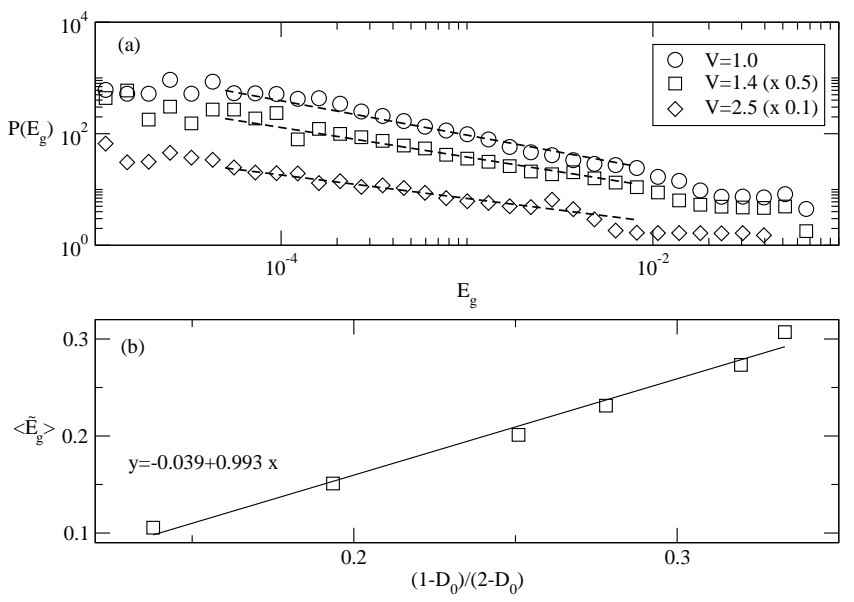

FIG. 3: (a) The distribution $\mathcal{P}\left(E_{1}\right)$ for hybrid structures where the normal sample is a Fibonacci lattice with $L=987$ and $M=1$ and various $V$ 's. The dashed lines are the theoretical predictions (2); (b) The mean value $\left\langle\tilde{E}_{1}\right\rangle$ versus the theoretical prediction (3) for various $V$ values. The straight line $y=-0.039+0.993 x$ represents the best linear fit.

practically not affected by the proximity of the system to the superconductor. Thus these eigenstates and the corresponding eigenenergies are solutions of the eigenvalue problem for the hybrid structure as well. We point here that the perturbative assumption used in our argument, was verified numerically in [15], where it was observed that the effect of the coupling of systems with fractal spectra to external leads is a small perturbation for the most of the eigenstates.

The distribution $\mathcal{P}\left(E_{1}\right)$ for the normal system can be derived in the following way. We scan the spectrum of the normal system with a set of Fermi energies which have resolution given by $\epsilon$. Then for fixed $E_{1}$ we count the number of Fermi energies that are in a distance $E_{1}$ with tolerance $\epsilon$ from the next larger eigenenergy of the normal system. This is given by the number of level spacings which are larger than $E_{1}$ i.e. $\int_{E_{1}}^{s_{\max }} p(s) d s$ where $s_{\max }$ is the maximum level spacing and $p(s)$ is the level spacing distribution. The normalized gap density in the limit of $\epsilon \rightarrow 0$ is then given by

$$
\mathcal{P}\left(E_{1}\right)=\frac{\int_{E_{1}}^{s_{\max }} p(s) d s}{\int_{0}^{s_{\max }} d E_{1} \int_{E_{1}}^{s_{\max }} p(s) d s}
$$

Substituting in the above equation the expression for $p(s)=s^{-\left(1+D_{0}\right)}$ we eventually get

$$
\mathcal{P}\left(\tilde{E}_{1}\right)=\left(\frac{1}{D_{0}}-1\right)\left(\tilde{E}_{1}^{-D_{0}}-1\right)
$$

where $\tilde{E}_{1}=\frac{E_{1}}{s_{\max }}$ is the rescaled energy gap. Notice that an additional factor 2 should be introduced in the definition of the rescaled energy gap i.e. $\tilde{E}_{1}=2 \frac{E_{1}}{s_{\max }}$ once we turn to the distribution of the hybrid structure, due to 


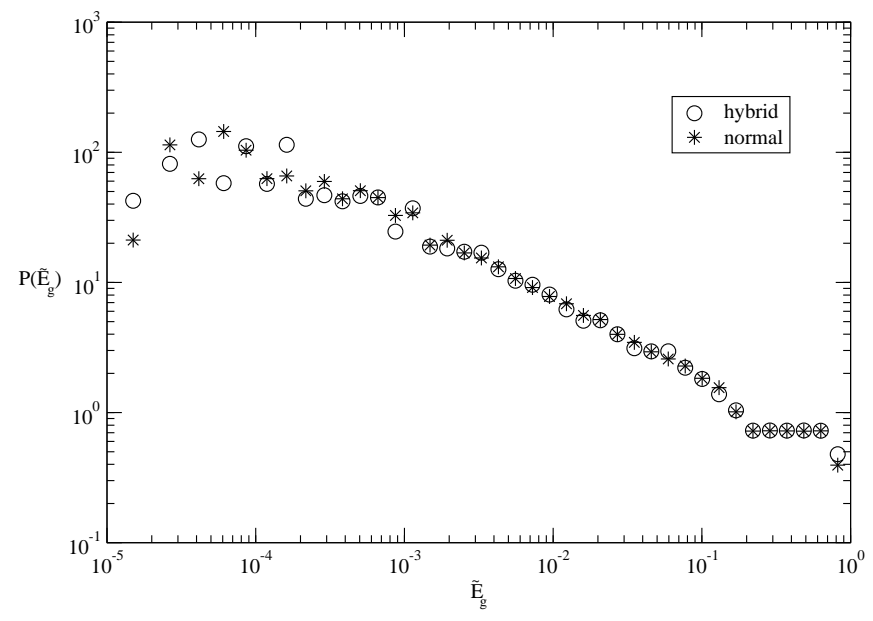

FIG. 4: The distribution $\mathcal{P}\left(\tilde{E}_{1}\right)$ for a hybrid structure where the normal part is a Fibonacci system with $L=987$ and $M=50$ and $V=1.4(\circ)$. Overplotted $(\star)$ is the corresponding distribution $\mathcal{P}\left(\tilde{E}_{1}\right)$ for the same Fibonacci system disconnected from a superconductor.

the fact that the excitation levels for the hybrid structure are coming in pairs. In Figs. 1 and 3a we plot with dashed lines the above theoretical prediction. Using Eq. (8) the mean $E_{1}$ and can be easily evaluated leading to Eq. (3).

In conclusion we have studied the statistical properties of the excitation spectrum of a normal system with fractal spectra in the proximity to a superconductor. We have found that the underlying classical dynamics is irrelevant and that the statistical properties of the quasispectrum depends only on the fractal nature of the normal system. Such a system can be realized by a twodimensional electron gas subject to a perpendicular magnetic field and periodic potential in contact with a superconductor [16]. It is known that the normal system of this type can be mapped onto the Harper model [7, 8, 14] possessing fractal spectrum at the critical point.

Finally, our results can be of interest for the quantum optics community with respect to studies of reflection of light by a dielectric medium in front of a phase-conjugation mirror [17. This problem is the optics analogue of Andreev reflection [18]. A wave incident at frequency $\omega_{0}+\Delta \omega$ is retro-reflected at frequency $\omega_{0}-\Delta \omega$ where $\omega_{0}$ is the pumped frequency of the phaseconjugation mirror. The analogue of $\omega_{0}$ and of the frequency shifts $\Delta \omega$ are the Fermi energy $E_{F}$ and the excitation energies $E$ respectively. The latter were shown here to be controlled by the fractal dimension $D_{0}$. Keeping in mind the above analogies it is reasonable to conjecture that $\Delta \omega$ is controlled as well by $D_{0}$ in quasi-periodic optical structures like e.g. in Fibonacci quasicrystals [19]. Thus controlling $D_{0}$ we can tune $\Delta \omega$ which was predicted in [17] to affect drastically the reflected intensity from a phase conjugation mirror for a certain parameter range.

It is our pleasure to thank J. Cserti, R. Fleischmann, C.
Lambert and H. Schomerus, for useful discussions. T.K. acknowledges the officers of the $5^{\text {th }} \mathrm{A}$ Artillery Unit for their support. This research was supported by a grant from the GIF, the German-Israeli Foundation for Scientific Research and Development.

[1] J. A. Melsen, P. W. Brouwer, K. M. Frahm and C. W. J. Beenakker, Europhys. Lett. 35, 7 (1996); Phys. Scrip. 69, 223 (1997); W. Ihra, M. Leadbeater, J. L. Vega and K. Richter, Eur. Phys. J. B 21, 425 (2001); K. M. Frahm, P. W. Brouwer, J. A. Melsen and C. W. J. Beenakker, Phys. Rev. Lett. 76, 2981 (1996); J. Cserti, A. Kormanyos, Z. Kaufmann, J. Koltai and C. J. Lambert, ibid. 89, 057001 (2002).

[2] M. G. Vavilov, P. W. Brouwer, V. Ambegaokar and C. W. J. Beenakker, Phys. Rev. Lett. 86, 874 (2001).

[3] Ph. Jacquod, H. Schomerus and C. W. J. Beenakker, Phys. Rev. Lett. 90, 207004 (2003).

[4] H. Schomerus and C. W. J. Beenakker, Phys. Rev. Lett. 82, 2951 (1999);

[5] P. M. Ostrovsky, M. A. Skvortsov and M. V. Feigel'man, Phys. Rev. Lett. 87, 027002 (2001); cond-mat/0202524 M. Hiller, A. Ossipov, T. Kottos and T. Geisel, in preparation (2003).

[6] C. E. Porter, Statistical Theory of Spectral Fluctuations, Academic Press, New York (1965); B. L. Altshuler and B. I. Shklovskii, Zh. Eksp. Teor. Fiz. 91, 220 (1986) [Sov. Phys. JETP 64, 127 (1986)].

[7] T. Geisel, R. Ketzmerick and G. Petschel, Phys. Rev. Lett. 66, 1651 (1991); T. Geisel, R. Ketzmerick and G. Petschel, in Quantum Chaos; Between Order and Disorder, J. Casati and B. Chirikov, eds. (Cambridge) 634-659.

[8] P. G. Harper, Proc. Roy. Soc. Lond. A68, 874 (1955); S. Aubry, G. Andre, Ann. Israel Phys. Soc. 3, 133 (1980); S. N. Evangelou, J.-L. Pichard, Phys. Rev. Lett. 84, 1643 (2000); F. Piéchon, Phys. Rev. Lett., 76 4372, (1996).

[9] D. Shechtman, I. Blech, D. Gratias, and J. W. Cahn, Phys. Rev. Lett. 53, 1951 (1984); M. Kohmoto, L. P. Kadanoff and C. Tang, Phys. Rev. Lett. 501870 (1983); J. M. Luck and D. Petritis, J. Stat. Phys. 42, 289 (1986); J. Bellisard, B. Iochum, E. Scoppola, and D. Testard, Comm. Math. Phys. 125, 527 (1989).

[10] P. Leboeuf, J. Kurchan, M. Feingold, and D. P. Arovas, Phys. Rev. Lett. 65, 3076 (1990); T. Geisel, R. Ketzmerick and G. Petschel, Phys. Rev. Lett. 67, 3635 (1991); R. Artuso, F. Borgonovi, I. Guarneri, L. Rebuzzini, and G. Casati, Phys. Rev. Lett. 69, 3302 (1992).

[11] R. Artuso, G. Casati and D. Shepelyansky, Phys. Rev. Lett. 68, 3826 (1992); R. Artuso, F. Borgonovi, I. Guarneri, L. Rebuzzini and G. Casati, ibid. 69, 3302 (1992).

[12] G. M. Zaslavskii, M. Yu. Zakharov, R. Z. Sagdeev, D. A. Usikov, A. A. Chernikov, JETP Letters 44, 451 (1986).

[13] P. W. Anderson, Phys. Rev. 109, 1492 (1958); A. MacKinnon and B. Kramer, Rep. Prog. Phys. 56, 1469 (1993).

[14] We have also checked the validity of Eqs. (23) for the Harper model at criticality described by Eq. (6) with $V_{n}=2 \cos (2 \pi \sigma n)$ and $\sigma=(\sqrt{5}+1) / 2$.

[15] A. Ossipov, M. Weiss, Tsampikos Kottos, and T. Geisel, Phys. Rev. B 64, 224210 (2001) 
[16] J. Eroms, M. Tolkiehn, D. Weiss, U. Roössler, J. De Boeck and G. Borghs, Europhys. Lett. 58, 569 (2002); I. E. Batov, Th. Schäpers and A. V. Ustinov in Physics of semiconductors 2002, Proceedings of the 26th ICPS conference, Edinburgh, ed. A.R. Long and J.H. Davies, (Institute of Physics Publishing, Bristol and Philadelphia); D. Uhlisch et. al., Phys. Rev. B 61, 12463 (2000); F. Rahman, et. al., ibid. 54, 14026 (1996).

[17] J. C. J. Paasschens, P. W. Brouwer and C. W. J.
Beenakker, Europhys. Lett. 38651 (1997); J. C. J. Paasschens, M. J. M. de Jong, P. W. Brouwer and C. W. J. Beenakker, Phys. Rev. A 56, 4216 (1997).

[18] We thank H. Schomerus for pointing this to us.

[19] L. Dal Negro, C. J. Oton, Z. Gaburro, L. Pavesi, P. Johnson, A. Lagendijk, R. Righini, M. Colocci, D. S. Wiersma, Phys. Rev. Lett. 90, 055501 (2003) 